\title{
ON LQG CONTROL OF LINEAR STOCHASTIC SYSTEMS WITH CONTROL DEPENDENT NOISE
}

\author{
John B. Moore \\ Department of Systems Engineering and \\ Cooperative Research Center for Robust and Adaptive Systems \\ Research School of Information Sciences and Engineering \\ Australian National University \\ Canberra A.C.T. 0200 \\ AUSTRALIA \\ John.Moore@anu.edu.au \\ Xunyu Zhou
}

Department of Systems Engineering and Engineering Management

The Chinese University of Hong Kong

Shatin N.T.

HONG KONG

xyzhou@se.cuhk.edu.hk

Andrew E.B. Lim

Department of Electrical \& Electronic Engineering

University of Melbourne

Parkville Victoria 3052

AUSTRALIA

andrewQee.mu.OZ.AU

\begin{abstract}
:
This paper announces initial results of studies on partially observed linear quadratic Gaussian (LQG) models where the stochastic disturbances depend on both states and controls and the measurements may be bilinear in the noise and the states/controls. While the Separation Theorem of standard LQG design does not apply in any strict sense, suboptimal linear state estimate feedback laws are derived based on certain linearizations. The controllers may well be useful for nonlinear stochastic systems where linearized models which include terms bilinear in the noise and states/controls are significantly more accurate
\end{abstract}

The original version of this chapter was revised: The copyright line was incorrect. This has been corrected. The Erratum to this chapter is available at DOI: 10.1007/978-0-387-35359-3_40 
than if these terms are set to zero. These controllers are calculated by solving a generalized discrete time Riccati equation. The properties of this equation relating to well posedness of the associated LQG problem are discussed.

\section{INTRODUCTION}

The classical linear quadratic Gaussian (LQG) control theory for stochastic linear systems assumes that the stochastic disturbances are additive and not control or state dependent $[2,3]$. Relaxing this assumption to allow linear state and control dependence in noise leads to a much broader class of stochastic models, which have applications in real-world systems (see [6] for more details). On the other hand, working with this class allows improved approximation by linearization of nonlinear stochastic systems than if the bilinear terms involving the noise are excluded. Recently, LQG theory has been generalized for such a class of linear/bilinear stochastic systems in continuous time [6,7]. Optimal state feedback control laws are linear and are calculated by solving a so-called stochastic matrix Riccati equation which specializes the familiar conventional LQG Riccati equations when the disturbances are independent of the states and controls. The stochastic Riccati equations are by no means as well understood as in the standard case, at least in the continuous time setting. There remains open questions concerning existence and uniqueness of the solutions of these equations. There is also an intriguing property that the control weighting matrix $R$ in a standard quadratic integral cost term need not be positive definite, even in the continuous time case. This property reveals some deep nature of uncertainty. For details see $[6,7]$.

What is the situation then for the partially observed case? To what extent does the standard LQG methodology [2] with its Separation Theorem apply? Can we achieve useful linear state estimate feedback laws?

In this paper the above questions are addressed for discrete time case and some initial thoughts and results are presented. The expectation is that since the models are bilinear in the state and the noise as well as in the control and the noise, some of the virtues of a standard linear Gaussian theory will be lost. Certainly, even if the noise signals are Gaussian, the states and control signals will be generally non-Gaussian. Consequently, optimal (information) state estimators will be infinite dimensional, in general, see for example [4]. Even so, since a conditional linear minimum square error (LMSE) covariance state estimator is known for the models of interest, and is finite dimensional, it makes sense from an implementation point of view to work with such a state estimator and a linear state estimate feedback law, even if such a law is suboptimal.

The conditional LMSE filter has the structure of a Kalman filter, see [2], but with a Kalman gain which is state estimate and control dependent. Likewise, the quadratic state cost, when expressed in terms of state estimates instead of true states, is nonlinear. Appropriate linearizations of the filter equations and cost terms, neglecting higher order terms but allowing terms bilinear in the noise and controls/state estimates in the filter, allows then application of a 
discrete-time analogy of the recently studied LQG theory in [6]. This leads to an 'optimal' linear state estimate feedback law under assumptions of negligible higher order terms. In practise, this law has some degree of sub-optimality because the neglected higher order terms may be significant to some extent. However, the neglected terms do not include terms bilinear in the innovations (prediction errors) and the state estimates/control, so there is a chance for improved performance over the standard LQG approach which neglects these terms as well as higher order terms.

The paper is organized as follows. In Section 2 an optimal feedback controller is derived for a completely observed discrete time LQG with state and control dependent noise. As in the standard case, solving a discrete time Riccati equation is a key step in calculating the optimal controller. In fact, the associated Riccati equation is a generalization of the standard discrete time Riccati equation. The existence properties of this equation and its relationship to the well posedness of the control problem is discussed in Section 3. Section 4 is concerned with an approximate Kalman filter for the partially observed LQG model. Finally, suboptimal linear state estimate feedback laws are obtained in Section 5 by combining the results in Sections 2 and 4 .

\section{DISCRETE TIME LQG RESULTS}

In this section, we derive discrete time versions of those in [6] which will be useful in a later section.

Consider the discrete time stochastic signal model

$$
x_{k+1}=\left(A_{k}+w_{k}^{A} \Delta A_{k}\right) x_{k}+\left(B_{k}+w_{k}^{B} \Delta B_{k}\right) u_{k}+w_{k}
$$

where $x_{k} \in \mathbf{R}^{n}$ is the state, $u_{k} \in \mathbf{R}^{m}$ is the control, and $w_{k}^{A}, w_{k}^{B} \in \mathbf{R}^{1}$ are noise terms, assumed here to be martingale increments on $\mathcal{G}_{k-1}$, where $\mathcal{G}_{k-1}$ is the $\sigma$-algebra generated by past noise terms up to $w_{k-1}^{A}, w_{k-1}^{B}, w_{k-1}$. The covariances are assumed to be

$$
E\left[\left(w_{k-1}^{A}\right)^{2} \mid \mathcal{G}_{k-1}\right]=E\left[\left(w_{k-1}^{B}\right)^{2} \mid \mathcal{G}_{k-1}\right]=1, \quad E\left[w_{k} w_{k}^{\prime} \mid \mathcal{G}_{k-1}\right]=Q_{k},
$$

and

$E\left[w_{k-1}^{A} w_{k-1}^{B} \mid \mathcal{G}_{k-1}\right]=\rho_{k}^{A B}, \quad E\left[w_{k-1} w_{k-1}^{A} \mid \mathcal{G}_{k-1}\right]=\rho_{k}^{A}, \quad E\left[w_{k-1} w_{k-1}^{B} \mid \mathcal{G}_{k-1}\right]=\rho_{k}^{B}$.

Generalizations of the dependent noise terms $w_{k}^{A} \Delta A_{k}$ and $w_{k}^{B} \Delta B_{k}$ to the case of non-scalar noise is immediate by working with terms $\sum_{i=1}^{N} w_{k}^{A^{i}} \Delta A_{k}^{i}$ and $\sum_{i=1}^{N} w_{k}^{B^{i}} \Delta B_{k}^{i}$.

The performance index of the problem is given by the standard quadratic sum cost

$$
J_{T}=E\left\{\sum_{k=0}^{T-1}\left(x_{k}^{\prime} Q_{k}^{c} x_{k}+u_{k}^{\prime} R_{k+1}^{c} u_{k}\right)+x_{T}^{\prime} Q_{T}^{c} x_{T}-x_{0}^{\prime} Q_{0}^{c} x_{0}\right\}
$$


In this model, all the $A_{k}, \Delta A_{k}$, etc.. are (deterministic) matrices with appropriate dimensions, $Q_{k}^{c}$ and $Q$ are non-negative definite matrices, and $R_{k}^{c}$ are symmetric matrices (could be indefinite, as in standard discrete time LQG theory).

Let us solve the above stochastic optimal control problem in two different cases. The results derived below will be applied in Section 4 for partially observed models.

Case I: $\rho_{k}^{A}=\rho_{k}^{B}=0$.

Let us consider first the case when $w_{k} \perp w_{k}^{A}, w_{k}^{B}$, so that $\rho_{k}^{A}=\rho_{k}^{B}=0$. In this case, the optimal control takes the form

$$
u_{k}=K_{k}^{c} x_{k}
$$

where

$$
\begin{aligned}
K_{k}^{c} & =-\left(\Omega_{k+1}^{c}\right)^{-1} L_{k+1}^{c}, \\
L_{k+1}^{c} & =B_{k}^{\prime} S_{k+1} A_{k}+\rho_{k}^{A B} \Delta B_{k}^{\prime} S_{k+1} \Delta A_{k}, \\
\Omega_{k+1}^{c} & =B_{k}^{\prime} S_{k+1} B_{k}+\Delta B_{k}^{\prime} S_{k+1} \Delta B_{k}+R_{k+1}^{c} .
\end{aligned}
$$

Here, $S_{k}$ is the solution of a backward matrix Riccati equation

$$
\left\{\begin{array}{l}
S_{k}=A_{k}^{\prime} S_{k+1} A_{k}-L_{k+1}^{c}{ }^{\prime}\left(\Omega_{k+1}^{c}\right)^{-1} L_{k+1}^{c}+\left(Q_{k}^{c}+\Delta A_{k}^{\prime} S_{k+1} \Delta A_{k}\right) \\
S_{T}=Q_{T}^{c}
\end{array}\right.
$$

By standard completion of squares arguements, it can be shown that (1)-(2) is well posed if $\Omega_{k+1}^{c}$ is positive definite. In this case, the control law (2)-(4) is the unique optimal control.

In continuous time LQG theory, it is generally required that $R$ is strictly positive definite for the problem to be well posed. Recent results by Chen, Li and Zhou [6] for the continuous time problem show that $R$ can have negative eigenvalues if the diffusion term in the system equations depend on the control. It is interesting to note therefore that in the discrete time problem, the control weighting matrices $R_{k}$ can have negative eigenvalues and the problem remain well posed, even if the bilinear terms $\Delta A_{k}$ and $\Delta B_{k}$ are all zero!

Case II: $\rho_{k}^{A} \neq 0, \rho_{k}^{B} \neq 0$.

In the event that $w_{k}$ and $\left(w_{k}^{A}, w_{k}^{B}\right)$ are correlated so that $\rho_{k}^{A}, \rho_{k}^{B} \neq 0$, then the optimal control requires not only the state feedback term as in (3) but also an external input as

$$
u_{k}=K_{k}^{c} x_{k}+b_{k}
$$

where $b_{k}$ is calculated by linear backward recursions. For details, the reader should consult [5].

\section{DISCRETE TIME RICCATI EQUATION}

In the continuous time LQG problem, a standard assumption is that $R(t)$ is strictly positive definite. In the paper by Chen, Li and Zhou [6], it is shown 
that this assumption is not necessary when the diffusion term depends on the control. In this section, we examine the effect of the terms $\Delta A_{k}$ and $\Delta B_{k}$ on the well posedness of the LQG problem (1)-(2).

Recall that the LQG problem (1)-(2) is well posed if and only if $\Omega_{k}^{c} \geq 0$ for every $k$. Note once again that it is possible for the standard LQG problem (ie. $\Delta A_{k}=0$ and $\Delta B_{k}=0$ ) to be well posed with either $Q_{k}<0$ or $R_{k}<0$ (but obviously not both). We show in this section that if $\Delta A_{k} \neq 0$ or $\Delta B_{k} \neq 0$, then $Q_{k}$ and $R_{k}$ can be made 'more negative' and with the associated problem still remaining well posed. Bounds on the allowable decrease are also derived for certain special cases.

Before doing this however, we need to introduce some notation. Let $\mathcal{K}=$ $\left\{\left(S_{0}, \cdots, S_{T}\right) \mid S_{j} \in \mathbf{R}^{n \times n}\right.$, symmetric $\}, \mathcal{Q}=\left\{\left(Q_{0}, \cdots, Q_{T}\right) \mid Q_{j} \in \mathbf{R}^{n \times n}\right.$, symmetric $\}$ and $\mathcal{P}=\left\{\left(R_{1}, \cdots, R_{T}\right) \mid R_{j} \in \mathbf{R}^{m \times m}\right.$, symmetric $\}$. Given a sequence $\bar{R}^{c}=$ $\left(\bar{R}_{1}^{c}, \cdots, \bar{R}_{T}^{c}\right) \in \mathcal{P}$ of control weights and $\bar{Q}^{c}=\left(\bar{Q}_{0}^{c}, \cdots, \bar{Q}_{T}^{c}\right) \in \mathcal{Q}$ of state weights, the standard discrete time Riccati equation

$$
\left\{\begin{aligned}
S_{k} & =A_{k}^{\prime} S_{k+1} A_{k}-A_{k}^{\prime} S_{k+1} B_{k}\left(\bar{R}_{k+1}^{c}+B_{k}^{\prime} S_{k+1} B_{k}\right)^{-1} B_{k}^{\prime} S_{k+1} A_{k}+\bar{Q}_{k}^{c} \\
S_{T} & =\bar{Q}_{T}^{c}
\end{aligned}\right.
$$

gives rise to a sequence $\left(S_{0}, \cdots, S_{T}\right) \in \mathcal{K}$. Hence, we can define a mapping $\psi: \mathcal{P} \times \mathcal{Q} \rightarrow \mathcal{K}$ which maps a sequence of control weights $\bar{R}^{c}=\left(\bar{R}_{1}^{c}, \cdots, \bar{R}_{T}^{c}\right) \in$ $\mathcal{P}$ and state weights $\bar{Q}^{c}=\left(\bar{Q}_{0}^{c}, \cdots, \bar{Q}_{T}^{c}\right) \in \mathcal{Q}$ to the solution $\psi\left(\bar{Q}^{c}, \bar{R}^{c}\right)=$ $\left(\psi_{0}\left(\bar{Q}^{c}, \bar{R}^{c}\right), \cdots, \psi_{T}\left(\bar{Q}^{c}, \bar{R}^{c}\right)\right) \in \mathcal{K}$ of $(6)$.

Suppose now that $\bar{Q}^{c}=Q^{c} \in \mathcal{Q}$ is given (and fixed) while $\bar{R}^{c}$ is the variable. In this case, we shall write $\psi\left(Q^{c}, \bar{R}^{c}\right)$ simply as $\psi\left(\bar{R}^{c}\right)$. It follows that the associated (standard) LQG problem is solvable if and only if

$$
\bar{R}_{k+1}^{c}+B_{k}^{\prime} \psi_{k+1}\left(\bar{R}^{c}\right) B_{k} \geq 0
$$

Our result for the case $\Delta A_{k}=0$ is as follows:

Theorem 1 Let $\left(Q_{k}^{c}, R_{k}^{c}\right)$ be given. If $\Delta A_{k}=0$, then the problem (1)-(2) corresponding to $\left(Q_{k}^{c}, R_{k}^{c}\right)$ is well posed if and only if

$$
R_{k+1}^{c} \geq \bar{R}_{k+1}^{c}-\Delta B_{k}^{\prime} \psi_{k+1}\left(\bar{R}^{c}\right) \Delta B_{k}
$$

for some $\bar{R}_{k}^{c}$ such that $\left(Q_{k}^{c}, \bar{R}_{k}^{c}\right)$ satisfies (6)-(7).

Note in particular that if $\Delta B_{k} \neq 0$, then the control weighting matrices $\bar{R}_{k}^{c}$ can be made 'more negative' and the problem (1)-(2) still remains well posed. The bound on this change is given by (8).

In Theorem 1 , we assume that $\bar{Q}^{c}$ is given and fixed. However, it can be shown that $\bar{Q}_{k}^{c}$ can be made 'more negative' if $\Delta A_{k}=0$ and $\Delta B_{k} \neq 0$ [5]. The allowable bounds on this change is still an open question.

Consider now the case when $\Delta A_{k} \neq 0$ but $\Delta B_{k}=0$. Let $\bar{R}^{c}=R^{c} \in \mathcal{P}$ be fixed. Let $\psi: \mathcal{Q} \rightarrow \mathcal{K}$ be a mapping such that $\psi\left(\bar{Q}^{c}\right)=\psi\left(\bar{Q}^{c}, R^{c}\right)$ is the 
solution of the standard discrete time Riccati equation

$$
\left\{\begin{array}{l}
S_{k}=A_{k}^{\prime} S_{k+1} A_{k}-A_{k}^{\prime} S_{k+1} B_{k}\left(R_{k+1}^{c}+B_{k}^{\prime} S_{k+1} B_{k}\right)^{-1} B_{k}^{\prime} S_{k+1} A_{k}+\bar{Q}_{k}^{c} \\
S_{T}=\bar{Q}_{T}^{c} .
\end{array}\right.
$$

In this case, the associated (standard) LQG problem is solvable if and only if

$$
R_{k+1}^{c}+B_{k}^{\prime} \psi_{k+1}\left(\bar{Q}^{c}\right) B_{k} \geq 0
$$

In much the same way as the case $\Delta A_{k}=0, \Delta B_{k} \neq 0$, the following result can be shown.

Theorem 2 Let $\left(Q_{k}^{c}, R_{k}^{c}\right)$ be given. If $\Delta B_{k}=0$, then the problem (1)-(2) corresponding to $\left(Q_{k}^{c}, R_{k}^{c}\right)$ is well posed if and only if

$$
Q_{k}^{c} \geq \bar{Q}_{k}^{c}-\Delta A_{k}^{\prime} \psi_{k+1}\left(\bar{Q}^{c}\right) \Delta A_{k}
$$

for some $\bar{Q}_{k}^{c}$ such that $\left(R_{k}^{c}, \bar{Q}_{k}^{c}\right)$ satisfies (9)-(10).

As in the case of Theorem 1, Theorem 2 shows how much 'more negative' the matrices $\bar{Q}_{k}^{c}$ can be made when $\Delta A_{k} \neq 0$ and $\Delta B_{k}=0$. It can also be shown that if $\Delta A_{k} \neq 0$ and $\Delta B_{k}=0, \bar{R}_{k}^{c}$ can be made 'more negative' [5]. The allowable bounds on this change is still an open question. Similarly, the effect of both $\Delta A_{k} \neq 0$ and $\Delta B_{k} \neq 0$ is still unresolved.

\section{STATE ESTIMATION}

In this section, we first define a partially observed signal model. Next, we apply the known Kalman filter theory to yield a linear minimum variance state estimator, which is then linearized further so that the filter is linear in the states and control, and bilinear in the innovations (prediction errors) and the states/controls.

Consider the following partially observed model:

$$
\left\{\begin{array}{c}
x_{k+1}=\left(A_{k}+w_{k}^{A} \Delta A_{k}\right) x_{k}+\left(B_{k}+w_{k}^{B} \Delta B_{k}\right) u_{k}+w_{k} \\
y_{k}=\left(C_{k}+w_{k}^{c} \Delta C_{k}\right) x_{k}+v_{k}
\end{array}\right.
$$

where $y_{k} \in \mathbf{R}^{p}$. Here $w_{k}^{c}, v_{k}$ are martingale increments, each orthogonal to $w_{k}^{A}$, $w_{k}^{B}, w_{k}$, and $E\left[v_{k} v_{k}^{\prime}\right]=R_{k}$.

Linear conditional minimum variance state estimator: Applying standard filtering results [2] yields the estimator

$$
\left\{\begin{aligned}
\hat{x}_{k+1} & =A_{k} \hat{x}_{k}+B_{k} u_{k}+K_{k}\left(\hat{x}_{k}, u_{k}\right) \nu_{k} \\
\nu_{k} & =y_{k}-C_{k} \hat{x}_{k}
\end{aligned}\right.
$$

where the gain $K_{k}\left(\hat{x}_{k}, u_{k}\right)$ is given in terms of a coupled matrix Riccati equation as follows

$$
K_{k}\left(\hat{x}_{k}, u_{k}\right)=L_{k} \Omega_{k}\left(\hat{x}_{k}, u_{k}\right)^{-1}
$$


with

$$
\begin{aligned}
L_{k} & =A_{k} \Sigma_{k} C_{k}^{\prime} \\
\Omega_{k}\left(\hat{x}_{k}, u_{k}\right) & =C_{k} \Sigma_{k} C_{k}^{\prime}+R_{k}+\Delta C_{k}\left(\Sigma_{k}+\hat{x}_{k} \hat{x}_{k}^{\prime}\right) \Delta C_{k}^{\prime}
\end{aligned}
$$

and

$$
\left\{\begin{aligned}
& \Sigma_{k+1}= A_{k} \Sigma_{k} A_{k}^{\prime}-L_{k} \Omega\left(\hat{x}_{k}, u_{k}\right)^{-1} L_{k}^{\prime}+Q_{k}+\Delta A_{k}\left(\Sigma_{k}+\hat{x}_{k} \hat{x}_{k}^{\prime}\right) \Delta A_{k}^{\prime} \\
&+\Delta B_{k} u_{k} u_{k}^{\prime} \Delta B_{k}^{\prime} \\
& \Sigma_{0}=E\left[x_{0} x_{0}^{\prime}\right]
\end{aligned}\right.
$$

Here $\hat{x}_{k}$ is the best linear estimate conditioned on $\mathcal{Y}_{k-1}$, the $\sigma$-algebra generated by $y_{0}, \cdots, y_{k-1}$, where best is in a minimum error variance sense. The associated conditional error covariance is $\Sigma_{k}=E\left[\left(x_{k}-\hat{x}_{k}\right)\left(x_{k}-\hat{x}_{k}\right)^{\prime} \mid \mathcal{Y}_{k-1}\right]$. Notice that the dependence of the noise on states and controls in our model (12) leads to an error covariance which depends on the past measurements (and controls), and in turn leads to a filter gain $K_{k}(\cdot, \cdot)$ which is dependent on the past measurements (and controls). Now this dependency of $K_{k}(\cdot, \cdot)$ on $\hat{x}_{k}, u_{k}$ is by no means affine, but in order to proceed to a control law based on the LQG theory of Section 2, we must linearize $K_{k}(\cdot, \cdot)$ in $\hat{x}_{k}$ and $u_{k}$.

A filter bilinear in the innovations: Consider a linearization of $K_{k}(\cdot, \cdot)$, via a Taylor expansion, for simplicity in the $p=1$ case

$$
K_{k}\left(\hat{x}_{k}, u_{k}\right)=K_{k}+K_{k}^{x} \hat{x}_{k}+K_{k}^{u} u_{k}+o\left(\left\|\hat{x}_{k}\right\|,\left\|u_{k}\right\|\right) .
$$

Neglecting the quadratic and higher order terms in $\hat{x}_{k}, u_{k}$ leads to an approximate filter

$$
\begin{aligned}
\hat{x}_{k+1} & \approx A_{k} \hat{x}_{k}+B_{k} u_{k}+\left(K_{k}+K_{k}^{x} \hat{x}_{k}+K_{k}^{u} u_{k}\right) \nu_{k} \\
& =\left(A_{k}+K_{k}^{x} \nu_{k}\right) \hat{x}_{k}+\left(B_{k}+K_{k}^{u} \nu_{k}\right) u_{k}+K_{k} \nu_{k}
\end{aligned}
$$

\section{STATE ESTIMATE FEEDBACK}

The approach taken in an LQG control design is taken here, namely to consider the state estimator (13) (or in our case the approximation (17)) as a state space signal model with state $\hat{x}_{k}$, and to re-organize the control performance index $J_{T}$ of (2) in terms of $\hat{x}_{k}$, rather than $x_{k}$. Noting (13) and the definition for $\Sigma_{k}$, we have a re-organization of $J_{T}$ as

$$
J_{T}=\sum_{k=0}^{T-1}\left[\hat{x}_{k}^{\prime} Q_{k}^{c} \hat{x}_{k}+u_{k}^{\prime} R_{k+1}^{c} u_{k}+\operatorname{tr}\left(Q_{k}^{c} \Sigma_{k}\right)\right] .
$$

Actually, $\Sigma_{k}$ is perhaps best written as $\Sigma_{k}\left(\hat{x}_{k} \hat{x}_{k}^{\prime}, u_{k} u_{k}^{\prime}\right)$ since it is dependent on $\hat{x}_{k} \hat{x}_{k}^{\prime}$ and $u_{k} u_{k}^{\prime}$. Now a Taylor Series expansion leads to

$$
\Sigma_{k} \approx \Sigma_{k}^{0}+\Sigma_{k}^{x} \hat{x}_{k} \hat{x}_{k}^{\prime}+\Sigma_{k}^{u} u_{k} u_{k}^{\prime},
$$


being linear in $\hat{x}_{k} \hat{x}_{k}^{\prime}$ and $u_{k} u_{k}^{\prime}$. Thus (18) under (19) becomes

$$
J_{T} \approx \sum_{k=0}^{T-1}\left[\hat{x}_{k}^{\prime}\left(Q_{k}^{c}+\Sigma_{k}^{x}\right) \hat{x}_{k}+u_{k}^{\prime}\left(R_{k+1}^{c}+\Sigma_{k}^{u}\right) u_{k}+\operatorname{tr}\left(Q_{k}^{c} \Sigma_{k}^{0}\right)\right]
$$

Now the optimization of (20) under (17) can be tackled using the optimal LQG results of Section 2 with $w^{A}=w^{B}=w$. Thus

$$
u_{k}^{o p t} \approx K_{k}^{c} \hat{x}_{k}+b_{k}
$$

where $\hat{x}_{k}$ is derived from the filter (17). Also $K_{k}^{c}$ are derived from an approximate specialization of (3)-(4) in which $\Delta A_{k}=K_{k}^{x}, \Delta B_{k}=K_{k}^{u}$. The term $b_{k}$ is derived by solving backward recursions for $b_{T}, b_{T-1}, \cdots, b_{0}[5]$.

\section{References}

[1] B.D.O. Anderson and J.B. Moore. Optimal Control: Linear Quadratic Methods. Prentice Hall, New Jersey, 1989.

[2] B.D.O. Anderson and J.B. Moore. Optimal Filtering. Prentice Hall, New Jersey, 1978.

[3] M.H.A. Davis. Linear Estimation and Stochastic Control Chapman and Hall, London, 1977.

[4] R.J. Elliott, L Aggoun and J.B. Moore. Hidden Markov Models: Estimation and Control. Springer-Verlag, 1995.

[5] J.B. Moore, X.Y. Zhou and A.E.B. Lim. Suboptimal LQG control of linear systems with control dependent noise. (submitted).

[6] S.P. Chen, X.J. Li and X.Y. Zhou. Stochastic linear quadratic regulators with indefinite control weight costs. SIAM J. Contr. Optim., to appear.

[7] A.E.B. Lim and X.Y. Zhou. Optimal stochastic LQR control with integral quadratic constraints and indefinite control weights. (preprint) 